INPLASY

PROTOCOL

To cite: Yang et al.

Comparative efficacy of

tonifying kidney on treatment

of mammary gland

hyperplasia: Systematic review

with network meta-analysis.

Inplasy protocol 202220049.

doi:

10.37766/inplasy2022.2.0049

Received: 13 February 2022

Published: 14 February 2022

Corresponding author:

Yang xujie

yangxujie2008@sina.com

Author Affiliation:

Hebei Univrsity of Chinese

Medicine.

Support: National Natural

Science Found.

Review Stage at time of this

submission: Data analysis.

Conflicts of interest:

None declared.

\section{Comparative efficacy of tonifying kidney on treatment of mammary gland hyperplasia: Systematic review with network meta-analysis}

\author{
Yang, XJ1; Pei, $\mathrm{XH}^{2}$.
}

Review question / Objective: What is the role of kidneytonifying therapy in the treatment of breast hyperplasia?

Condition being studied: Breast hyperplasia is a common and frequently-occurring disease in women. Its incidence is closely related to various factors such as mental factors, menstrual cycle, and estrogen secretion disorders. The general health, social function and psychological health indicators of patients with breast hyperplasia are seriously damaged. At present, the estrogen receptor antagonist tamoxifen is used to treat hyperplasia of mammary glands at home and abroad. This drug causes some patients to experience decreased or delayed menstruation, as well as gastrointestinal symptoms such as nausea and vomiting. The treatment of this disease in traditional Chinese medicine is mostly based on syndrome differentiation and treatment, and adopts the prevention and treatment methods such as resolving phlegm and dissipating stagnation, promoting blood circulation and removing blood stasis, and softening firmness and dissipating stagnation. Among them, using the relationship between the liver and the kidney in the theory of traditional Chinese medicine, taking nourishing the kidney yin and kidney yang as the starting point to prevent and treat mammary gland hyperplasia has a significant effect.

INPLASY registration number: This protocol was registered with the International Platform of Registered Systematic Review and Meta-Analysis Protocols (INPLASY) on 14 February 2022 and was last updated on 14 February 2022 (registration number INPLASY202220049).

\section{INTRODUCTION}

Review question / Objective: What is the role of kidney-tonifying therapy in the treatment of breast hyperplasia?
Condition being studied: Breast hyperplasia is a common and frequentlyoccurring disease in women. Its incidence is closely related to various factors such as mental factors, menstrual cycle, and 
estrogen secretion disorders. The general health, social function and psychological health indicators of patients with breast hyperplasia are seriously damaged. At present, the estrogen receptor antagonist tamoxifen is used to treat hyperplasia of mammary glands at home and abroad. This drug causes some patients to experience decreased or delayed menstruation, as well as gastrointestinal symptoms such as nausea and vomiting. The treatment of this disease in traditional Chinese medicine is mostly based on syndrome differentiation and treatment, and adopts the prevention and treatment methods such as resolving phlegm and dissipating stagnation, promoting blood circulation and removing blood stasis, and softening firmness and dissipating stagnation. Among them, using the relationship between the liver and the kidney in the theory of traditional Chinese medicine, taking nourishing the kidney yin and kidney yang as the starting point to prevent and treat mammary gland hyperplasia has a significant effect.

\section{METHODS}

Participant or population: Research on humans, regardless of age, gender or country.

Intervention: Studies of all types of interventions will be included.

Comparator: Controls will include active and placebo.

Study designs to be included: This study will include randomised controlled trials (RCT).

Eligibility criteria: This study will include randomised controlled trials (RCT).

Information sources: This study was searched through PubMed, Cochrane Library, Embase, World Sci-Net, Allied and Complementary Medicine Database (AMED), Web of Science, China Knowledge Infrastructure (CNKI), Chongqing VIP China Science and Technology Journal Database (VIP), CNKI and Wanfang database.
Main outcome(s): Main ending: Total effective rate, effective rate of ablation, effective rate of pain relief, tumor diameter, pain threshold score; Secondary ending: Estradiol, Prolactin, Progesterone, Follicle Stimulating Hormone, Luteinizing Hormone, Testosterone.

Quality assessment / Risk of bias analysis: The Cochrane Collaboration's risk of bias tool will be used to assess the methodological quality of eligible RCTs. The tool focuses on selection bias (random sequence generation and allocation concealment), performance bias (blinding of participants and personnel), detection bias (blinding of outcome assessment), churn bias (incomplete outcome data), perstudy Selective reporting bias and other biases. Two independent reviewers will use this tool to assess the quality of randomized controlled trials. Any disagreements between the two reviewers will be resolved by discussion or the introduction of a third reviewer.

Strategy of data synthesis: Data for statistical analysis will be extracted into an excel file. Hazard ratios with $95 \%$ Cls will be used to investigate dichotomous data. For continuous outcomes, data will be analyzed using standard mean differences (SMD) and $95 \%$ Cls or weighted mean differences ( WMD). SMD will be used for different assessment tools. Frequency framework-based network meta-analyses will be performed using the net-meta package in $\mathbf{R}$ software (www.r-project.org, version 3.2.0), integrating direct and indirect evidence from the included randomized controlled trials. A network diagram will be drawn to show the number and interrelationships of interventions, as well as direct comparisons between interventions. Intervention outcomes will be ranked according to their P-values. Concordance will be assessed by implementing Cochran's Q statistic.

Subgroup analysis: Subgroup analyses will be performed to identify potential clinical and statistical heterogeneity and inconsistency. This includes patient age, 
sex and disease duration, trial blinding, quality of evidence, etc. A meta-regression analysis will be performed to quantify differences between subgroups and explore statistical significance.

Sensitivity analysis: A sensitivity analysis will be performed in this study.

Language: English.

Country(ies) involved: China.

Keywords: systematic review, network meta-analysis, mammary gland hyperplasia, tonifying kidney.

Contributions of each author:

Author 1 - Yang Xujie.

Email: yangxujie2008@sina.com

Author 2 - Pei Xiaohua.

Email: medicalhistory@sina.com 